\title{
Multiple myeloma with intracranial extension and bilateral renal infiltration: A case report and review of the literature
}

\author{
MIN-JUAN ZHANG ${ }^{1}$, GUO-HONG SU ${ }^{1}$, JIE SHEN ${ }^{1}$, FENG-HAI LIU ${ }^{2}$, YAN-FENG XU ${ }^{2}$, XIAO-LING ZHANG ${ }^{3}$, \\ QING WANG $^{1}$, RUI-HUAN WANG ${ }^{1}$, CHUN-YAN LIU $^{1}$ and YU-LEI ZHAO ${ }^{1}$ \\ ${ }^{1}$ Second Department of Haematology; Departments of ${ }^{2}$ Radiology and ${ }^{3}$ Pathology, Cangzhou Central Hospital, \\ Cangzhou, Hebei 061001, P.R. China
}

Received September 22, 2014; Accepted July 7, 2015

DOI: $10.3892 / \mathrm{ol} .2015 .3567$

\begin{abstract}
Multiple myeloma (MM) is a rare hematological malignancy, characterized by uncontrolled proliferation of plasma cells in the bone marrow. MM is usually confined to the bone marrow, however, it may occasionally infiltrate other tissues, which is known as extramedullary plasmacytoma (EMP). The majority of EMPs involve the head and neck region, although different anatomical sites, including the gastrointestinal tract, central nervous system, thyroid gland and breast may also be affected. The simultaneous presentation of EMP in the kidney and head is rare, presenting diagnostic challenges due to its unusual location and non-specific or absent symptoms. To the best of our knowledge, no case of extramedullary plasmacytoma presenting with simultaneous renal and intracranial infiltration has been reported in the literature thus far. However, the present study reports a case of primary renal and intracranial extramedullary plasmacytoma in a 76-year-old male patient. The patient presented with a swelling over the right side of the forehead, which had slowly increased in size prior to hospital admission. The swelling was associated with dizziness and weakness, without bone pain. Contrast magnetic resonance imaging suggested an osteolytic skull lesion with intracranial extension. Abdominal enhanced computed tomography scanning revealed a large tumor mass extending around and into the kidneys. Immunohistochemical examination of the renal tumor biopsy, and blood and serum samples, as well as immunoelectrophoresis of serum proteins, resulted in a diagnosis of EMP being proposed. Therefore, the patient was administered with two cycles of cyclophosphamide and thalidomide in combination with dexamethasone. Follow-up imaging performed 4 months later revealed almost complete disappearance of the intracranial tumor mass and
\end{abstract}

Correspondence to: Miss. Min-Juan Zhang, Second Department of Haematology, Cangzhou Central Hospital, 201 Xinhua Road, Cangzhou, Hebei 061001, P.R. China

E-mail: zhmj1981@126.com

Key words: multiple myeloma, extramedullary plasmacytoma, intracranial extension, renal infiltration, chemotherapy renal infiltration. The current study also presented a review of the literature. This study revealed that EMPs may co-exist with MM or present as the main symptom of MM. The diagnosis of an EMP is complex and requires radiological, hematological, biochemical and histological investigation. At present, no guidelines for EMP treatment have been established and thus, treatment options include surgery, chemotherapy and radiotherapy, either alone or in combination. We hypothesize that combined treatment may provide the best patient outcome.

\section{Introduction}

Multiple myeloma ( $\mathrm{MM})$, or myelomatosis, is a rare type of hematological malignancy that comprises $\sim 10 \%$ of all hematological malignancies. It is a neoplastic proliferation characterized by the uncontrolled proliferation of plasma cells in the bone marrow that leads to bone osteolytic destruction, anemia, recurrent infections and renal impairment. The incidence of MM is $~ 3-4$ per 100,000 individuals (1). Common presenting features of MM include bone pain and bone fractures, anemia, increased susceptibility to infections, hypercalcemia, neurological manifestations, hyperviscosity syndrome and renal failure $(1,2)$.

Although MM is usually confined to the bone marrow, it may occasionally infiltrate into other tissues $(3,4)$, and this is known as extramedullary plasmacytoma (EMP). To the best of our knowledge, there are no recommended guidelines for the treatment of EMP. Treatment options include surgery, chemotherapy and radiotherapy, either alone or combination (5). Local radiotherapy is the preferred therapeutic modality for EMP owing to its documented radiosensitivity (6). However, previous studies indicated that a combination treatment may provide the best results (7). The majority of EMPs involve the head and neck regions (8), although different anatomical sites, including the gastrointestinal tract, central nervous system, thyroid gland and breast may also be affected (7). However, it is rare that EMPs present initially with simultaneous intracranial and renal infiltration.

The current study reports a case of primary intracranial and renal plasmacytoma in a 76-year-old male patient. Written informed consent was obtained from the patient's family and ethical approval was obtained from the committee of Cangzhou Central Hosptial (Cangzhou, China). 
A

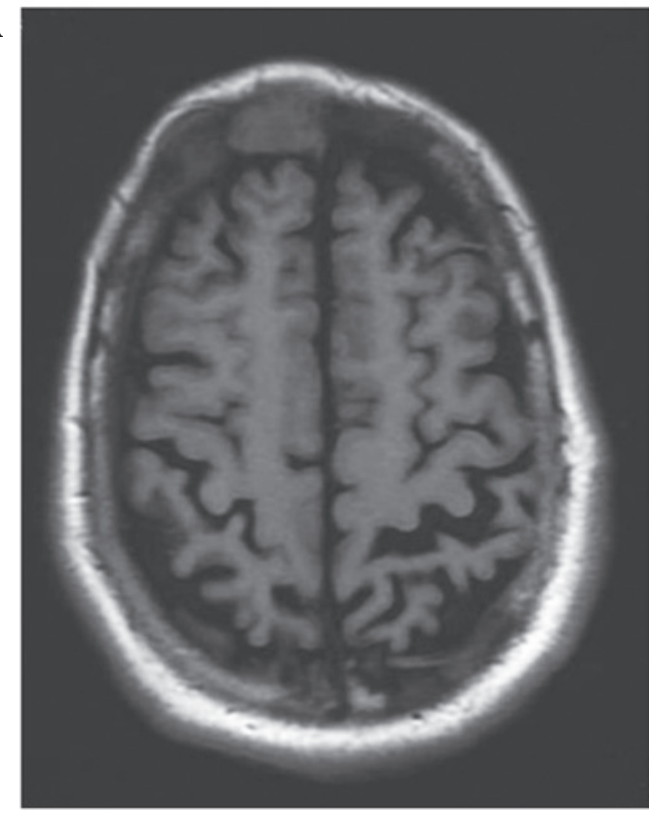

B

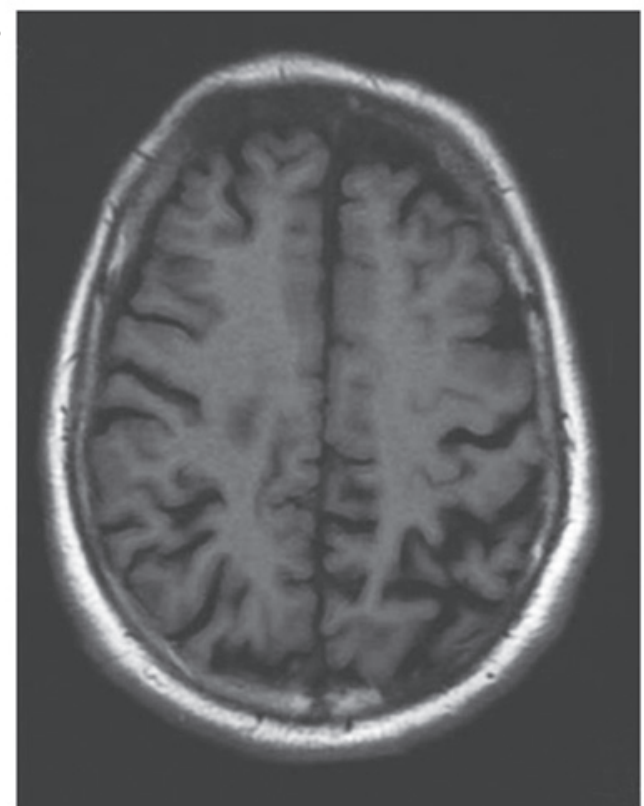

Figure 1. (A) MRI scan identified a tumor located in the right frontal part of the skull with intracranial expansion. (B) MRI scan performed following treatment with chemotherapy demonstrating that the tumor mass had practically disappeared. MRI, magnetic resonance imaging.
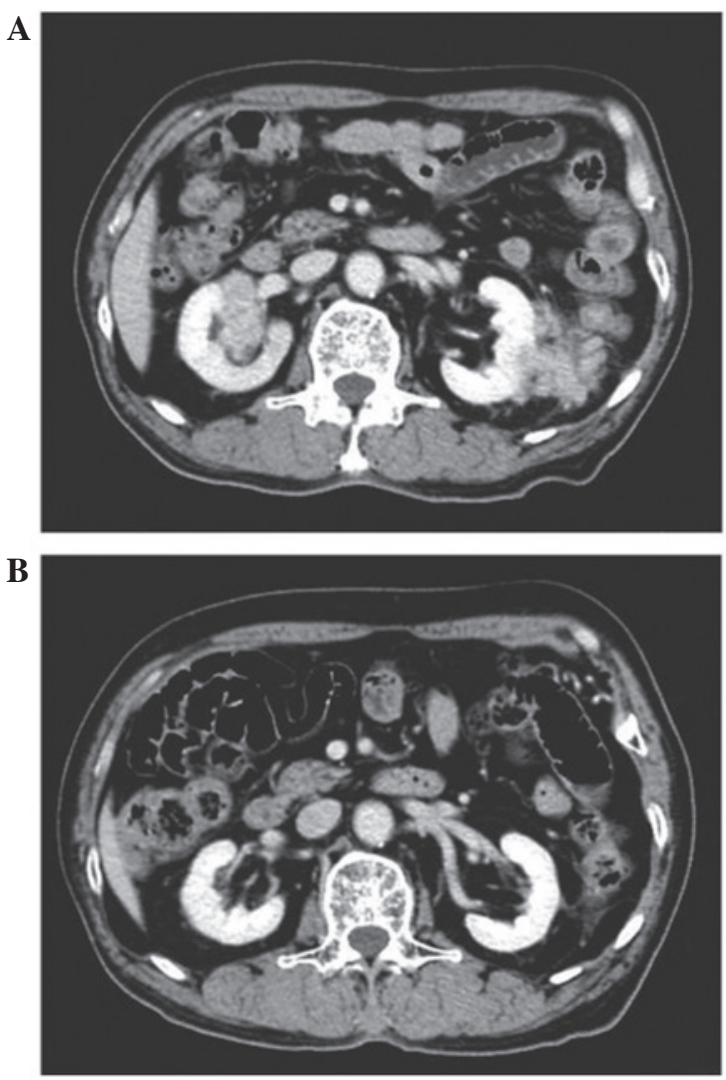

Figure 2. (A) Enhanced CT scans captured upon presentation revealed that the tumor mass infiltrated bilateral kidneys. (B) CT scans performed following treatment with chemotherapy demonstrated that the tumor masses had disappeared completely. CT, computed tomography.

\section{Case report}

The patient presented to the Cangzhou Central Hospital in
March 2014, complaining of swelling over the right side of the forehead, which had been slowly progressing during the 3 months prior to the date of admission, and was associated with dizziness and weakness. The patient did not present bone pain or a tendency for abnormal bleeding, and physical examination excluded lymphadenopathy and organomegally. A tense globular swelling of $5.0-6.5 \mathrm{~cm}$ in size was observed over the right frontal area, adhered to the underlying skull and skin. Contrast magnetic resonance imaging (MRI) suggested an osteolytic skull lesion with intracranial extension (Fig. 1A). Abdominal enhanced computed tomography (CT) scanning revealed a large tumor mass extending around and into the kidneys (Fig. 2A). Thus, a right renal biopsy was performed, and histopathological analysis of the tumor sample confirmed it to be plasmacytoma. Immunohistochemical staining was performed using cluster of differentiation (CD)38 and CD138 antibodies purchased from Beijing Xiya Jinqiao Biological Technology Co., Ltd. (Beijing, China). All stains were completed using a BenchMark machine (serial no. 815132; Ventana Medical Systems, Inc., Tucson, AZ, USA) and images were captured with an Olympus BX41 microscope (Olympus, Tokyo, Japan) and Image-Pro Plus software (Media Cybernetics, Inc., Rockville, MD, USA). Immunohistochemistry indicated that the tumor cells were positive for CD38 and CD138 (Fig. 3). The blood count demonstrated a hemoglobin concentration of $108 \mathrm{~g} / 1$, a white cell count of $6.07 \times 10^{9}$ cells/1 with no abnormal cells and a platelet count of $143 \times 10^{9}$ cells $/ 1$. The levels of serum creatinine, calcium and phosphorus were within the normal range (NR). The erythrosedimentation rate was $113 \mathrm{~mm} / \mathrm{h}$, and the level of serum $\beta 2$ microglobulin was 4,499 $\mu \mathrm{mol} / 1$ (NR, 0.9-2.7 $\mu \mathrm{mol} / \mathrm{l})$. The total serum protein concentration was $83 \mathrm{~g} / 1$ (NR, 60-83 g/l), and the levels of immunoglobulin (Ig)G, A and $\mathrm{M}$ were $39.25,3.01$ and $0.56 \mathrm{~g} / \mathrm{l}$, respectively (NR, 7.0-16.0, 0.7-4.0 and 0.4-2.3 g/l, respectively). Immunoelectrophoresis of serum proteins indicated the presence of an $\operatorname{IgG}$ paraprotein, and urine was positive for 
A

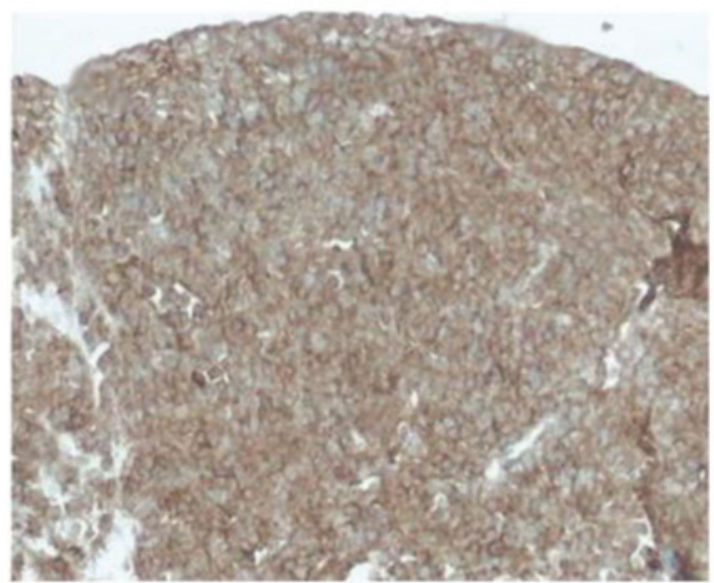

B

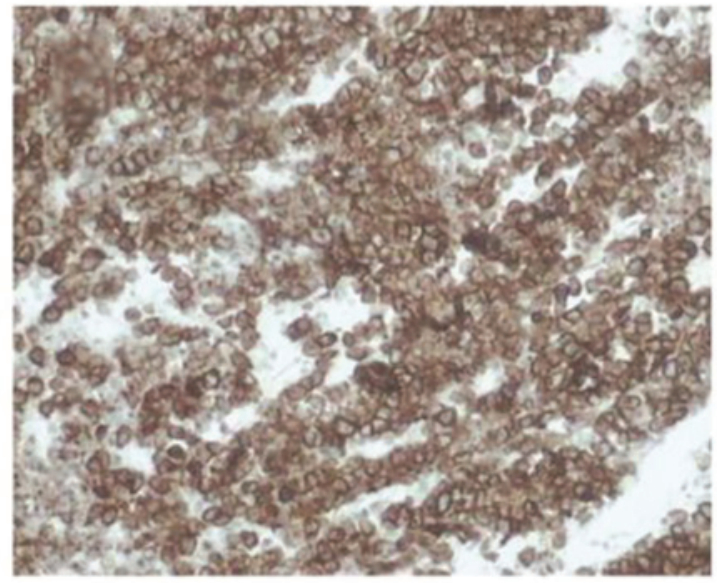

Figure 3. Immunohistochemical analysis of the infiltrate indicated strongly positive staining for (A) CD38 (magnification, x40) and (B) CD138 (magnification, $\mathrm{x} 100)$. CD, cluster of differentiation.

Bence-Jones (monoclonal) protein, indicating a diagnosis of MM (9). Bone marrow aspiration revealed $4 \%$ restriction of plasma cells, and whole body bone emission CT demonstrated multiple abnormal distribution of tracer concentration. Consequently, a diagnosis of MM was established.

The patient and his relatives refused further examination, surgical operation and radiotherapy, due to the patient's age and body condition. Therefore, the patient was subjected to chemotherapy, and was administered 2 cycles of cyclophosphamide and thalidomide in combination with dexamethasone. Follow-up imaging 4 months later revealed nearly complete disappearance of the intracranial tumor mass (Fig. 1B) and renal infiltration (Fig. 2B). The clinical condition also improved, and the patient experienced a complete recovery.

\section{Discussion}

EMP is a rare malignant neoplasm that develops due to uncontrolled plasma cell proliferation and monoclonal plasmacytic infiltration (10). EMPs may coexist with MM, presenting as the main symptom, developing during the course of MM, or occasionally occurring as isolated tumors $(11,12)$. Intracranial extramedullary plasmacytomas account for $4 \%$ of all primary plasma cell tumors (3). In patients with a diagnosis of MM, the presence of intracranial EMPs, as a result of the metastatic spread of $\mathrm{MM}$, is rare, occurring in $1 \%$ of all the reported cases $(13,14)$. To the best of our knowledge, $<30$ cases of renal plasmacytoma have been reported in the literature previously (15). In the cases of renal EMPs, the tumor mass is mostly confined to this anatomical area, and $<10 \%$ involve other organs besides the kidney $(16,17)$. Thus, to the best of our knowledge, the initial presentation of an EMP displaying intracranial and renal infiltration simultaneously, such as in the present case, has not been previously described.

The diagnosis of EMP is more complicated than that of $\mathrm{MM}$, and requires radiological, hematological, biochemical and histological examinations. Due to the absence of typical clinical symptoms and evidence from specific laboratory tests, a diagnosis of EMP may be delayed or missed, with potentially serious consequences for the patient. In the present case, EMP was diagnosed based on the clinical symptoms of the patient, alongside the imaging findings and the results of the serum Ig electrophoresis and the histopathological analyses of tissue biopsy and bone marrow aspirate.

To date, there are no specific guidelines for the treatment of EMP presenting with MM. The current treatment options for EMP include surgery, chemotherapy and radiotherapy, either alone or in combination $(3,15)$. If the tumor is located in an area with restricted surgical access, a complete resection with fractionated radiotherapy at the tumor site may be a good option for intracranial plasmacytomas. However, a number of case reports have highlighted the use of systemic chemotherapy and radiotherapy as the most important part of the treatment $(18,19)$. Recent studies on the fundamental molecular mechanisms responsible for MM cell growth and survival have led to the introduction of novel classes of pharmacological agents, including immunomodulatory drugs and proteasome inhibitors (20). In the present case, combined chemotherapy was selected as the treatment for EMP, due to the patient's age and body condition. In this context, chemotherapy may be an attractive option for certain cases, for example when the treatment is aimed at improving the quality of life of patients rather than at increasing the survival rate, particularly in elderly patients.

In conclusion, the simultaneous presentation of EMP in the kidney and head is rare. Thus, the present case report may aid in the diagnosis and treatment of future cases of EMPs.

\section{References}

1. Kyle RA, Gertz MA,Witzig TE,Lust JA,Lacy MQ, Dispenzieri A, Fonseca R, Rajkumar SV, Offord JR, Larson DR et al: Review of 1027 patients with newly diagnosed multiple myeloma. Mayc Clin Proc 78: 21-33, 2003.

2. Barlogie B, Epstein J, Selvanavagam P and Alexanian R: Plasma cell myeloma - new biological insights and advances in therapy. Blood 73: 865-879, 1989.

3. Cerase A, Tarantino A, Gozzetti A, Muccio CF, Gennari P, Monti L, Di Blasi A and Venturi C: Intracranial involvement in plasmacytomas and multiple myeloma: A pictorial essay. Neuroradiology 50: 665-674, 2008.

4. Turhal N, Henehan MD, Kaplan KL: Multiple myeloma: A patient with unusual features including intracranial and meningeal involvement, testicular involvement, organomegaly, and plasma cell leukemia. Am J Hematol 57: 51-56, 1998.

5. Kanoh T, Katoh H, Izumi T, Tsuji M and Okuma M: Renal plasmacytoma. Rinsho Ketsueki 34: 1470-1473, 199 (In Japanese).

6. Chao MV, Gibbs P, Wirth A, et al: Radiotherapy in the management of solitary extramedullary plasmacytoma. Inter Med J 35: 211-215, 2005.

7. Alexiou C, Kau RJ, Dietzfelbinger H, Kremer M, Spiess JC, Schratzenstaller B and Arnold W: Extramedullary plasmacytoma: Tumor occurrence and therapeutic concepts. Cancer 85: 2305-2314, 1999. 
8. Reed V, Shah J, Medeiros LJ, Ha CS, Mazloom A, Weber DM, Arzu IY, Orlowski RZ, Thomas SK, Shihadeh F, et al: Solitary plasmacytomas: outcome and prognostic factors after definitive radiation therapy. Cancer 117: 4468-4474, 2011.

9. Kyle RA, Larson DR, Therneau TM, Dispenzieri A, Melton LJ III, Benson JT, Kumar S and Rajkumar SV: Clinical course of lightchain smouldering multiple myeloma (idiopathic Bence Jones proteinuria): A retrospective cohort study. Lancet Haematol 1: e28-e36, 2014.

10. Laso FJ, Tabernero MD and Iglesias-Osma MC: Extramedullary plasmacytoma: A localized or systemic disease? Ann Intern Med 128: 156,1998

11. Jancelwicz Z, Takatsuki K, Sugai S amd Pruzanski W: IgD multiple myeloma. Review of 133 cases. Arch Intern Med 135: 87-93, 1975.

12. Dimopoulos MA, Kiamouris C and Moulopoulos LA: Solitary plasmacytoma of bone and extramedullary plasmacytoma. Hematol Oncol Clin North Am 13: 1249-1257, 1999.

13. Fassas AB, Muwalla F, Berryman, Benramdane R, Joseph L, Anaissie E, Sethi R, Desikan R, Siegel D, Badros A, et al: Myeloma of the central nervous system: Association with high-risk chromosomal abnormalities, plasmablastic morphology and extramedullary manifestations. Br J Haematol 117: 103-108, 2002.

14. Nieuwenhuizen L and Biesma DH: Central nervous system myelomatosis: Review of the literature. Eur J Haematol 80 $1-9,2008$.
15. Zhang SQ, Dong P, Zhang ZL, Wu S, Guo SJ, Yao K, Li YH, Liu ZW, Han H, Qin ZK, et al: Renal plasmacytoma: Report of a rare case and review of the literature. Oncol Lett 5: 1839-1843, 2013.

16. Solomito VL, and Grise J: Angiographic findings in renal (extramedullary) plasmacytoma. Case report. Radiology 102: 559-560, 1972.

17. Catalona WJ and Biles JD III: Therapeutic considerations in renal plasmacytoma. J Urol 111: 582-598, 1974.

18. Bindal AK, Bindal RK, van Loveren $H$ and Sawaya $R$ : Management of intracranial plasmacytoma. J Neurosurg 83: 218-221, 1995.

19. Gozzetti A, Cerase A, Lotti F, Rossi D, Palumbo A, Petrucci MT, Patriarca F, Nozzoli C, Cavo M, Offidani M, et al; GIMEMA (Gruppo Italiano Malattie Ematologiche dell'Adulto) Myeloma Working Party: Extramedullary intracranial localization of multiple myeloma and treatment with novel agents: A retrospective survey of 50 patients. Cancer 118: 1574-1584, 2012.

20. Raab MS, Podar K, Breitkreutz I, Richardson PG and Anderson KC: Multiple myeloma. Lancet 374: 324-339, 2009. 